\title{
Modulation Depth Enhancement for Randomly Arranged Tags in Passive RFID Tag to Tag Communications
}

\author{
Tarik Lassouaoui \\ Univ Lyon, INSA Lyon, Inria, CITI, EA3720 \\ 69621 Villeurbanne, France \\ tarik.lassaouaoui@insa-lyon.fr
}

\author{
Florin Hutu \\ Univ Lyon, INSA Lyon, Inria, CITI, EA3720 \\ 69621 Villeurbanne, France \\ florin-doru.hutu@insa-lyon.fr
}

\author{
Guillaume Villemaud \\ Univ Lyon, INSA Lyon, Inria, CITI, EA3720 \\ 69621 Villeurbanne, France \\ guillaume.villemaud@insa-lyon.fr
}

\author{
Yvan Duroc \\ Univ Lyon, Université Claude Bernard Lyon 1 \\ INSA Lyon, Ecole Centrale de Lyon, CNRS, Ampère, UMR5505 \\ Villeurbanne, France \\ yvan.duroc@univ-lyon1.fr
}

\begin{abstract}
In this paper, the performance evaluation of the tag to tag (T2T) communications using UHF (Ultra High Frequency) passive RFID (Radio Frequency Identification) tags is tackled. The T2T communication system is performed directly between a reader tag (RT) and a listener tag (LT) in the presence of an external electromagnetic (EM) field. Consequently, this kind of communication system implies the use of low RF signal levels, which makes it very sensitive to the noise, to the arrangement between the tags (i.e. spacing, tilt and shift) but also to the position of the source. The performance evaluation of this communication system is based on the study of the modulation depth, an indicator of the separation between the two modulation states and, indirectly, of the T2T communication quality. Considering scenarios in which the two tags are in the same plane but with the RT tilted and staggered with respect to the LT, our study demonstrates that the modulation depth can be kept higher for different separations between the tags by choosing the proper angle of incidence of the RF source with respect to the tag's plane. The proposed approach is studied through electromagnetic simulations and the obtained results are confronted with measurements.
\end{abstract}

Index Terms-backscattering, passive tags, UHF RFID, tag to tag communications.

\section{INTRODUCTION}

The RFID (Radio Frequency IDentification) is a constantly developing technology having numberless applications as logistics, access control, real-time localization or sensor networks [1]. Moreover, the development of the IoT (Internet of Things) requires ultra-low power or even battery free devices (sensor, actuators, etc.) and the RFID is an excellent candidate to reduce the energy footprint of the radio communications part. Particularly, the use of an external RF signal in order to carry at a short distance the information between two devices (backscatter principle) can be implemented by the means of RFID [2]. Nowadays, the RFID tags sensitivity remains the key issue since this parameter is defining the communication range.
The concept of tag to tag (T2T) communication has been introduced in [3]. Here, the authors demonstrates experimentally that two parallel passive or semi-passive tags closely spaced can directly communicate even in the absence of an RFID reader. The main idea is to establish a communication between a reader tag (RT) and a listener tags (LT) closely spaced with a reliable communication distance of about 25 millimetres. The envisaged T2T application scenarios demand to increase the communication range. Among these applications, a simple and powerless system allowing the personal protective equipment (PPE) usage monitoring may be implemented. The communication between the tags attached to each safety equipment can only be established when their relative positions have a certain configuration. By verifying the communication between tags, potential misalignment or negligence of the user can be avoided. Consequently, the full understanding of the mutual coupling among the tags and of the intrinsic electromagnetic (EM) characteristics of the antennas is necessary.

The T2T communication performance is dependent of the tag's relative position [4], [5]. Furthermore, the close communication range between tag's antennas brings up an important electromagnetic coupling. Consequently, the tag's antennas characteristics such as the radiation pattern or the input impedance are modified. These alterations have an impact on the T2T's communication performance since the modulation depth is potentially reduced.

To the best of our knowledge, few works have been done about this topic to improve this communication range. In [6], a simulation framework has been proposed as a tool allowing the performance evaluation of T2T radio links in terms of Bit Error Rate (BER). The analytical formulation of the probability of error in the case of a 2-ASK (Amplitude Shift Keying) modulation for coherent and non-coherent LT's receivers are given. In [7], the authors proposed a power efficient receiver working at modulation depth higher than $75 \%$ which was 


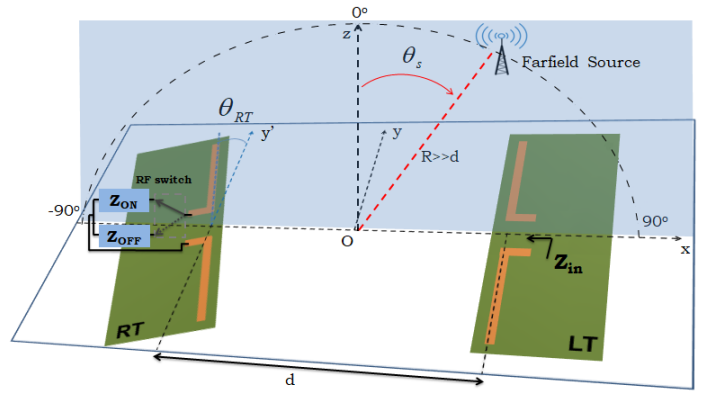

Fig. 1. Geometrical configuration of two printed dipoles, RT's antenna is spaced and tilted with respect to LT.

experimentally validated.

In this context, our study focuses on the impact of the different tag's arrangements (e.g. tilt or shift) which impacts the antenna's intrinsic electromagnetic characteristics with a direct consequence on the modulation depth. This study will be supplemented by electromagnetic simulations results and the measurements obtained with printed dipoles. Moreover, the constructive or destructive impact of the orientation of the external source with respect to the two tags is also studied. The rest of the paper is organized as follows. In Section II, the tag's antennas mutual coupling phenomena is described in order to study the LT's input impedance. In Section III, results are exploited in order to express the modulation depth and to study it for different T2T communication scenarios (spacing, tilt and stagger). Finally, the conclusion and the perspectives of this work are given in Section IV.

\section{LISTENER TAG'S IMPEDANCE BEHAVIOR IN PASSIVE T2T COMMUNICATIONS}

As stated, a T2T communication system operates at short distance, making it very sensitive to the tags configuration (i.e. spacing, tilt or shift). The electromagnetic coupling between the closely spaced antennas modifies their intrinsic electromagnetic characteristics such as radiation pattern and realised impedance.

The close interaction between the two tags is such that they have to be considered as an array of two coupled antennas characterized by its impedance matrix. The reader tag communicates by switching its load on an Open Circuit (OC) or a Short Circuit (SC). Depending on the RT's load, the LT tag's IC (integrated circuit) observes a particular value of the input impedance $Z_{\text {in }}$ according to the tags relative position. It is worth noting here that the gain of the two coupled antennas is also depending on the position of the source which can be a classical RFID reader or any other source providing a power level high enough to activate the tags (source is considered in far field).

\section{A. Theoretical T2T antenna's mutual coupling}

Two passive tags are placed in close vicinity one to each other. Each tag has an identical infinitely thin dipole antenna designed such that its intrinsic impedance (i.e. the impedance when the antenna is in free space) is $Z_{11}=50 \Omega$.

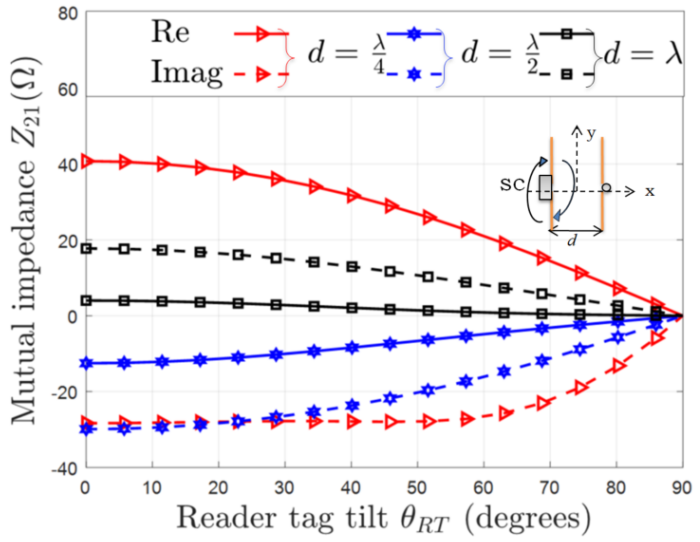

Fig. 2. Real and imaginary part of the mutual impedance $Z_{21}$ as function of $\theta_{R T}$ tilt angle of the RT's thin dipole for different distances $d$.

The currents induced in the two dipoles are assumed to be sinusoidal and at a frequency of $868 \mathrm{MHz}$. The RT's antenna is positioned at $\left(x_{R T}, y_{R T}\right)$ and its tilt angle with respect to $y$ axis is $\theta_{R T}$. The distance $d$ between the two tags is considered as fractions of the wavelength.

Fig. 2 presents the mutual impedances $Z_{21}$ of two coupled thin dipoles as a function of RT's antenna tilt angle $\theta_{R T}$ for different spacing distances $\frac{\lambda}{4}, \frac{\lambda}{2}$ and $\lambda$ respectively. The theoretical results are calculated by applying the IEMF (Induced Electromotive Force) method [8]. As expected, the spacing between the antennas and their tilt change significantly the mutual coupling. The mutual coupling decreases until it is canceled while the distance $d$ between the two antennas and the tilt angle $\theta_{R T}$ increases.

\section{B. Listener tag's input impedance}

To study the impact of the tags configuration (spacing, tilt and stagger) on the modulation depth $D$, simulations using CST Microwave studio were performed on a system of two closely coupled dipoles as shown in Fig. 1. The dipoles were $134 \mathrm{~mm} \times 1 \mathrm{~mm}$ copper traces on FR4 material (substrate dimensions $150 \mathrm{~mm} \times 10 \mathrm{~mm}$ ). The dimensions of the antennas have been calculated so as to have a low power reflected from the antenna $S_{11} \leq-22 \mathrm{~dB}$ at $868 \mathrm{MHz}$. These simulations are confronted with measurement results using a VNA (Vector Network Analyzer) with the same setup as in Fig. 1.

The input impedance $Z_{i n}$ is measured and compared with simulation results for various configurations. The two closely coupled tags are seen as a matrix of two antennas which are considered identical. Assuming the reciprocity between the two tag's identical antennas, we obtain $Z_{12}=Z_{21}$ and $Z_{11}=Z_{22}$.

In the two RT's loads $\left(Z_{L, O N}\right.$ and $\left.Z_{L, O F F}\right)$, the input impedance of the listener tag $Z_{\text {in }}$ can be written as follows:

$$
Z_{\text {in }, O N / O F F}=Z_{11}-\frac{Z_{21} Z_{12}}{Z_{22}+Z_{L, O N / O F F}}
$$




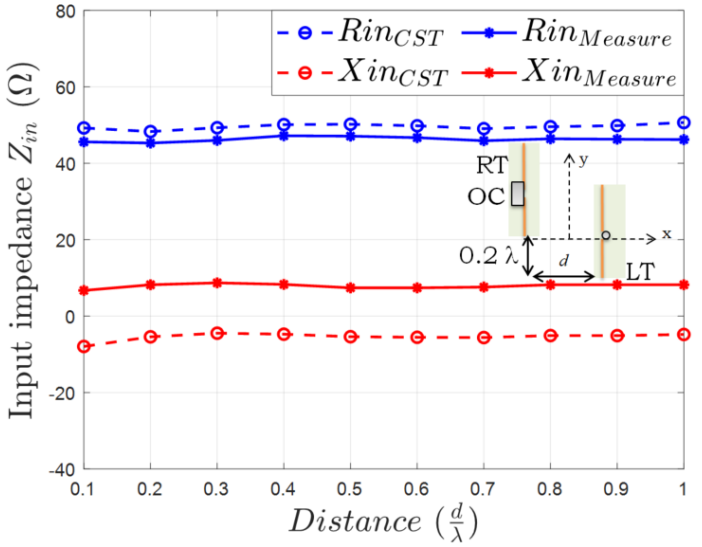

Fig. 3. Comparison between the simulation results with measurements for two tags one staggered by $0.2 \lambda$. Input resistance $\left(R_{i n}\right)$ and reactance $\left(X_{i n}\right)$ as function of distance $d$ between the tags when the RT tag is open circuited.

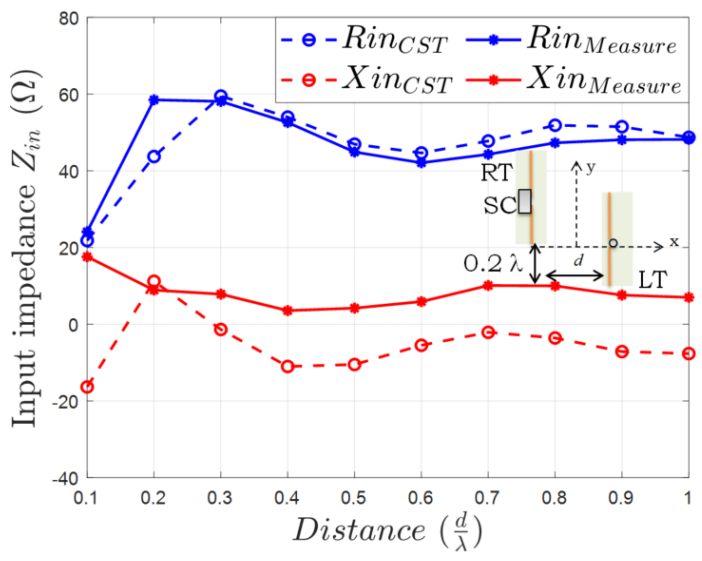

Fig. 4. Comparison between the simulation results with measurements for two tags one staggered by $0.2 \lambda$. Input resistance $\left(R_{i n}\right)$ and reactance $\left(X_{i n}\right)$ as function of distance $d$ between the tags when the RT tag is short circuited.

The real and imaginary parts of the LT's input impedance $Z_{i n}$ are presented in Fig. 3, 4 and 5, 6 for different configurations: one with RT staggered and then tilted respectively. The $Z_{i n}$ impedance is given as a function of the distance $d$ between the antennas and with respect to the two RT's commutation states. As can be seen from Fig. 4 and 6, the presence of RT's short circuited antenna modifies the input impedance of the LT's antenna. Moreover, we can remark the good agreement between the measurement and the EM simulations. The difference between the measured and simulated values is small, more precisely $\Delta R_{i n, S C \& O C}<2.7 \Omega$ and $\triangle X_{i n, S C \& O C}<9.8 \Omega$.

\section{ANALYSIS OF THE MODULATION DEPTH}

The T2T communication requires an RF source (dedicated or not) with sufficient power to ensure tags activation. In our study, we considered a source that provides a continuous Wave $(\mathrm{CW})$. The external RF source orientation $\theta_{s}$ is considered relative to the $z$ axis as shown in Fig. 1. Due to the commutation state of RT, the amplitude of the signal

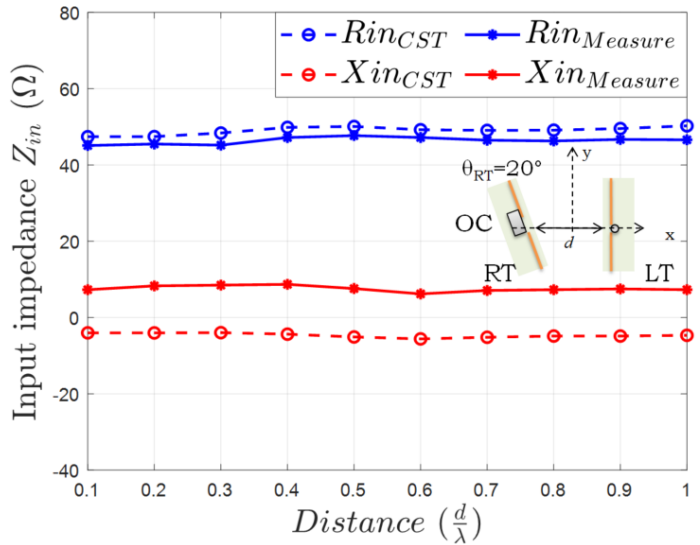

Fig. 5. Comparison between the simulation results with measurements for two tags one tilted by $\theta_{R T}=20^{\circ}$. Input resistance $\left(R_{i n}\right)$ and reactance $\left(X_{i n}\right)$ as function of distance $d$ between the tags when the RT tag is open circuited.

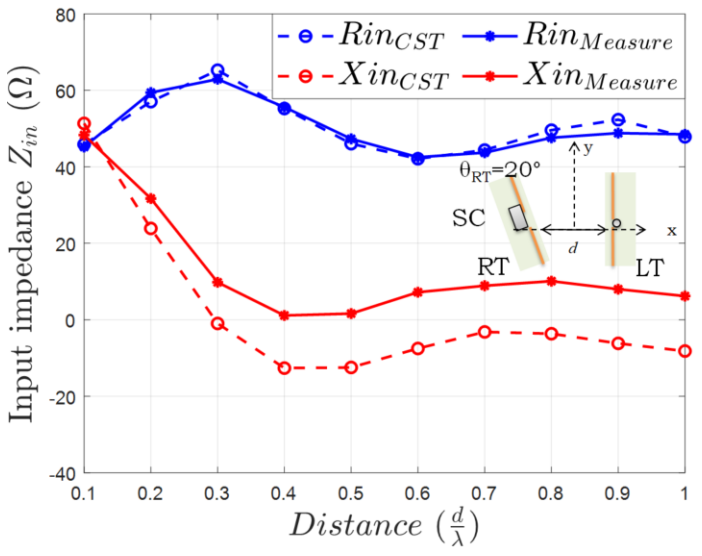

Fig. 6. Comparison between the simulation results with measurements for two tags one tilted by $\theta_{R T}=20^{\circ}$. Input resistance $\left(R_{i n}\right)$ and reactance $\left(X_{i n}\right)$ as function of distance $d$ between the tags when the RT tag is short circuited.

at the LT tag's input changes between two levels $A_{O N}$ and $A_{O F F}$. These parameters are defined by the LT's antenna input impedance $Z_{i n, O N / O F F}$ and the overall gain of the two antennas $G_{O N / O F F}$ in the direction of the considered farfield source, as shown in (2).

$$
A_{\text {ON/OFF }}=A_{\text {in }} \cdot G_{\text {ON/OFF }}\left|1-\left(\frac{Z_{\text {in, ON/OFF }}-Z_{0}}{Z_{\text {in, ON/OFF }}+Z_{0}}\right)\right|
$$

where $A_{i n}$ is the input voltage at $L T$ and $G_{O N / O F F}$ are the voltage gains and $Z_{0}$ characteristic impedance. Starting from these two amplitude levels $A_{O N}$ and $A_{O F F}$, the modulation depth $D$ is defined as in (3):

$$
D=\frac{\left|A_{O N}-A_{O F F}\right|}{\max \left(A_{O N}, A_{O F F}\right)} \cdot 100[\%]
$$

This parameter is taken as an indicator of the T2T communication quality. Indeed, the greater the modulation depth 


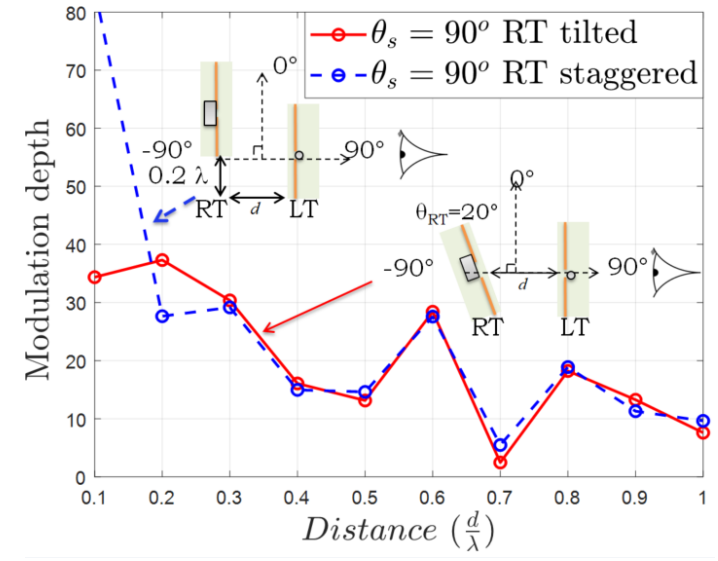

Fig. 7. Modulation depth $D$ at LT as function of the distance $d$ between tags. RT is tilted by $\theta_{R T}=20^{\circ}$ and RT tag staggered by $0.2 \lambda$, the source is on LT side $\theta_{s}=90^{\circ}$.

is, the easier is for the LT's baseband circuit to distinguish between the two states of the 2-ASK modulation.

As stated, the two tags relative position together with the external source position have an influence on the modulation depth $D$. In order to study this influence, a first scenario where RT tag is tilted by $\theta_{R T}=20^{\circ}$ and a second scenario where RT tag is staggered by $0.2 \lambda$ were simulated by considering the RF source placed perpendicular to the plane containing the two tags. As can be seen from Fig. 7, the maximum modulation depth is obtained for short distance between the tags. Globally, the modulation depth decreases when the two tags are separated.

In Fig. 7, the modulation depth for different distances between the two tags is given once when the RT tag is tilted by $20^{\circ}$ and then when RT tag is staggered by $0.2 \lambda$. The results show the importance of having a parallel arrangement even if RT is staggered in order to maximize the modulation depth. Unlike the tilted case, the staggered arrangement for short separation distances does not have a negative impact on the modulation depth, the coupling in this case being reinforced $(D=84 \%$ at $d=0.1 \lambda)$, thus facilitating the T2T communication.

The position of the source has also an effect on the modulation depth. In Fig. 8, the modulation depths for different source orientation $\left(\theta_{s}\right)$ are given. The incident angle of the source can be used to enhance the modulation depth $D$ depending on the separation distance between the tags. Therefore, it is important to know which is the best source position relative to the tag plane that maximizes the modulation depth.

\section{CONCLUSION}

Considering a passive $\mathrm{T} 2 \mathrm{~T}$ communication, this paper investigated the impact of the mutual coupling between the tags, their relative position and the source orientation, notably on the modulation depth. Indeed, higher the modulation depth is, more efficient the T2T communication is in terms of detection and noise robustness. Besides the relative arrangement of tags that modifies input impedances and radiation properties, the

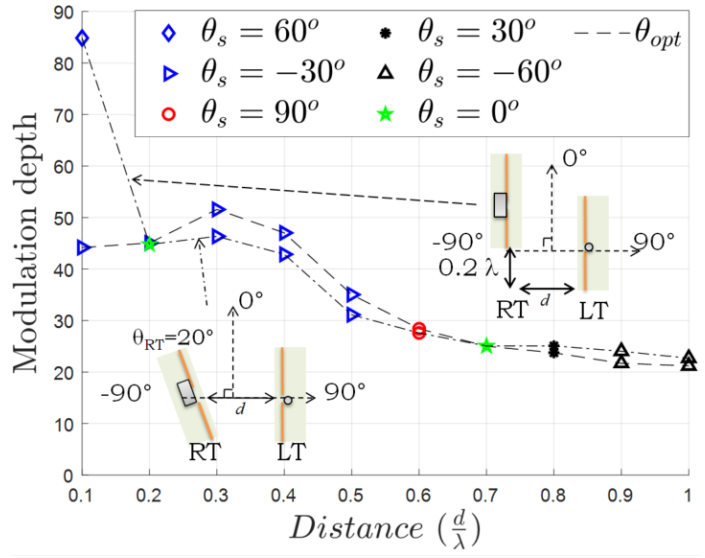

Fig. 8. Modulation depth $D$ enhancement by orientation of the source $\theta_{s}$ as function of the distance $d$ between tags. $D_{\max }=51.57 \%$ at $d=0.3 \lambda$ and $D_{\min }=21.21 \%$ at $d=\lambda$.

orientation of the RF source is also a critical parameter to take into consideration. The presented results describe the variation of the modulation depth for various scenarios. In consequence, with the objective of implementing a tag to tag system, our study would allow predict the T2T communication performance or establish specification guidelines.

In this study, only simple load impedances have been considered (short and open circuits). In perspective, a promising way would be to exploit complex load impedances in order to improve the modulation depth, and so the potential performance of the T2T communications.

\section{ACKNOWLEDGMENT}

This work was supported by the SPIE ICS-INSA Lyon IoT chair.

\section{REFERENCES}

[1] K. Finkenzeller, RFID handbook: fundamentals and applications in contact-less smart cards and identification. New York, NY, USA: John Wiley and Sons, Inc., 2003, p. 427.

[2] P. V. Nikitin, K. V. S. Rao, and S. Lam, "RFID paperclip tags," in 2011 IEEE International Conference on RFID, 2011, pp. 162-169.

[3] P. V. Nikitin, S. Ramamurthy, R. Martinez, and K. V. S. Rao, "Passive tag-to-tag communication," in 2012 IEEE International Conference on RFID (RFID), April 2012, pp. 177-184

[4] P. V. Nikitin and K. V. S. Rao, "Performance limitations of passive UHF RFID systems," in 2006 IEEE Antennas and Propagation Society International Symposium, July 2006, pp. 1011-1014.

[5] G. Marrocco and S. Caizzone, "Electromagnetic models for passive tagto-tag communications," IEEE Transactions on Antennas and Propagation, vol. 60, no. 11, pp. 5381-5389, 2012.

[6] T. Lassouaoui, F. Hutu, Y. Duroc, and G. Villemaud, "Theoretical BER evaluation of passive RFID tag-to-tag communications," in 2020 IEEE Radio and Wireless Symposium (RWS), 2020, pp. 213-216.

[7] Y. Karimi, A. Athalye, S. R. Das, P. M. Djurić, and M. Stanaćević, "Design of a backscatter-based tag-to-tag system," in 2017 IEEE International Conference on RFID (RFID), May 2017, pp. 6-12.

[8] H. Baker and A. LaGrone, "Digital computation of the mutual independance between thin dipoles," IRE Transactions on Antennas and Propagation, vol. 10, no. 2, pp. 172-178, 1962. 